\title{
Use of a Fast WDS Instrument for Identification of Minor EDS Peaks.
}

\author{
John Konopka
}

\section{Thermo Fisher Scientific}

The Electron Probe Micro Analyzer (EPMA) has been well established as providing the best possible resolution in x-ray microanalysis for over fifty years. These instruments and most of the Wavelength Dispersive Spectrometers (WDS) built for installation on SEMs employ a focusing geometry ${ }^{1}$. A different WDS instrument is now available that employs an x-ray optic used to focus x-rays from a sample onto a flat resolving crystal. Mechanically this device can be adapted to nearly any SEM port that can accommodate an EDS detector.

EDS is excellent for identifying major peaks in a spectrum. The relatively poor energy resolution of EDS makes reliable peak identification difficult for minor peaks or for small peaks overlapped by larger peaks. Using WDS as a complement to an EDS analyzer combines the strengths of both technologies. EDS can acquire data from all elements in a sample in one acquisition. When needed, WDS can be applied just to the narrow range of energies where EDS data has not resolved a peak well enough for identification.

Employing direct drive motors and lacking gears and belts this device can scan through x-ray energies very quickly. This high speed and the sensitivity of the x-ray optic combine to yield fast energy scans even in cases of low signal intensity, as is often encountered in the SEM.

Figure 1. shows a challenging peak identification problem. This could be $\mathrm{Mg} \mathrm{Ka}$, As La or a combination. Figure 2. shows this resolved by a WDS energy scan. Even with relatively little data the high resolution of WDS unambiguously reveals the presence of both Mg and As. Figure 3. shows the identification of a trace of $\mathrm{Cu}$ La under a dominant $\mathrm{Ni}$ La peak. In semiconductors it is important that these elements not be present together. Because of the small feature size it is necessary to use low $\mathrm{kV}$ for the analysis which requires the use of these L lines. Not only must the overlap be identified but the absence of $\mathrm{Cu}$ must be confirmed. Figure 4. shows the identification of $\mathrm{Hf} \mathrm{Ma} \mathrm{line.} \mathrm{The} \mathrm{sample} \mathrm{was} \mathrm{a}$ few nanometers of Hf deposited on GaAs. Figure 5. shows the identification of a nanoparticle of Ir on a silicon oxide glass bead. The small size of the particle necessitated the use of a FESEM and low beam current. In spite of the low signal intensity the analysis had to be done quickly or the beam would drift off of the sample. Even so, the identification of Ir is unambiguous.

All WDS data were acquired with a Thermo Scientific Magnaray WDS and NS7 EDS analyzer. The SEMs used are identified with each displayed spectrum. The fast, non-focussing geometry WDS instrument brings the high resolving power of WDS to the SEM/EDS analyst. Simplicity of operation, sensitivity and high speed remove barriers to adoption making it possible for most operators to have access to a technology which till now was restricted to more complicated, dedicated instruments.

References:

[1] KFJ Heinrich, “Electron Beam X-ray Microanalysis”, (Van Nostrand, New York) p.104. 


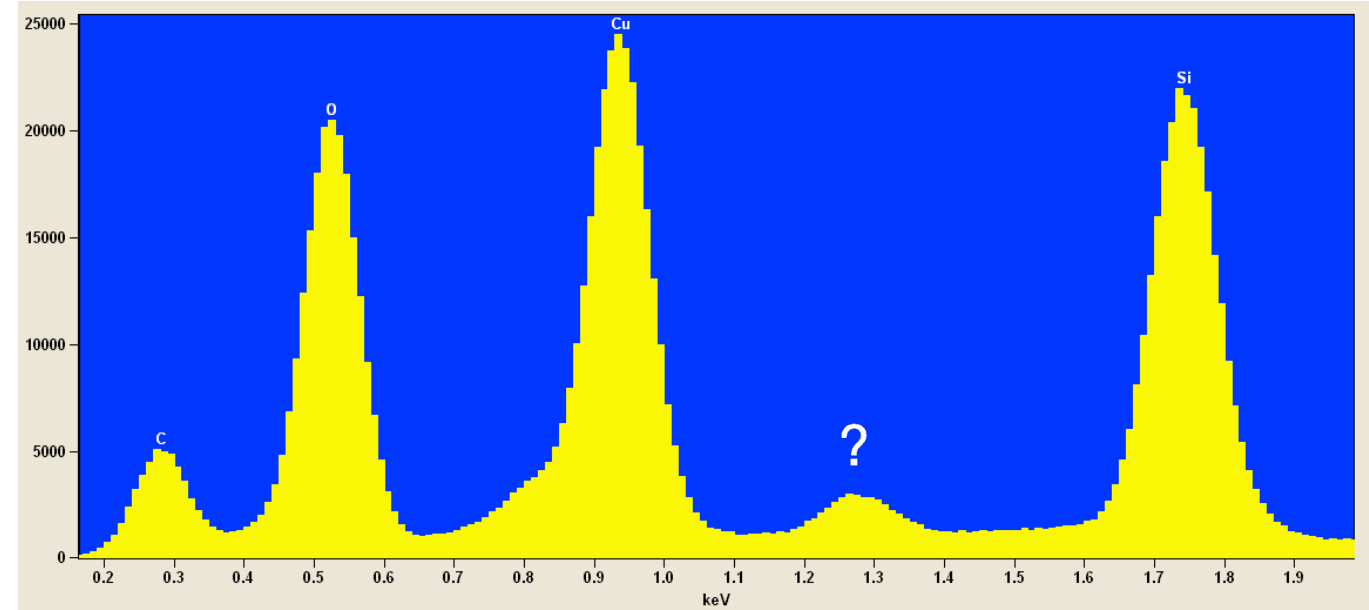

Figure 1. EDS spectrum of guanacoite bearing rock. Note the unknown peak at about $1.28 \mathrm{keV}$. This is resolved in Figure 2.

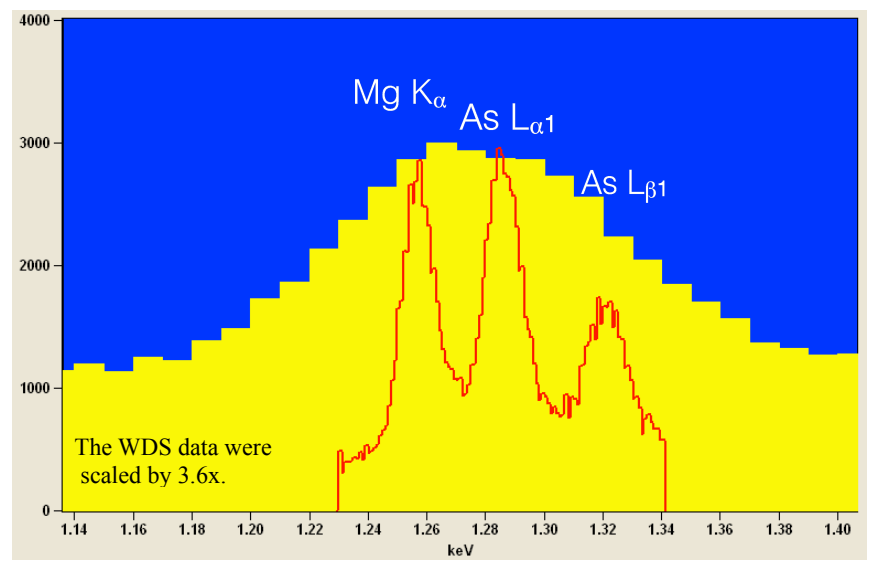

Figure 2. Identification of $\mathrm{Mg}$ and As. 1100s acquisition time. JEOL JSM-7100F FESEM.

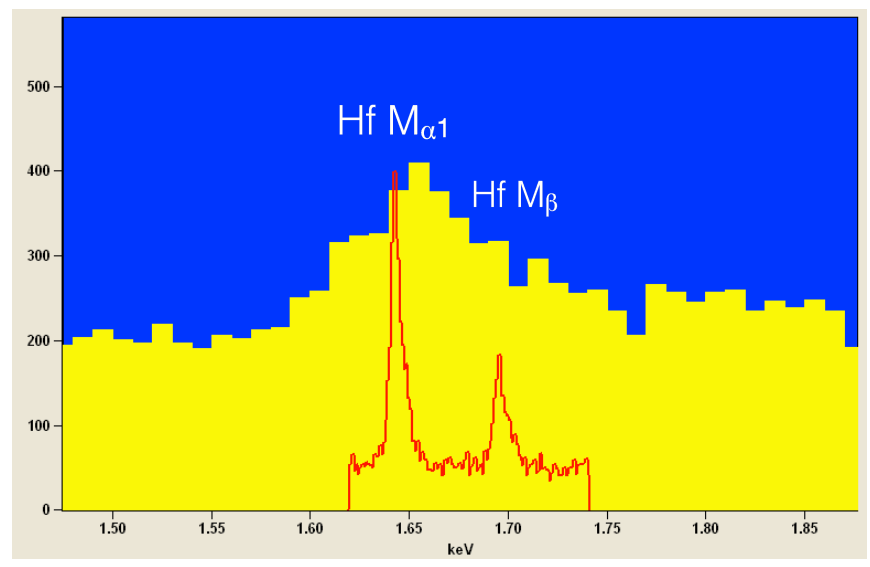

Figure 4. Identification of $\mathrm{Hf}$ film on GaAs. 363s acquisition time. JEOL JSM-7001F FESEM.

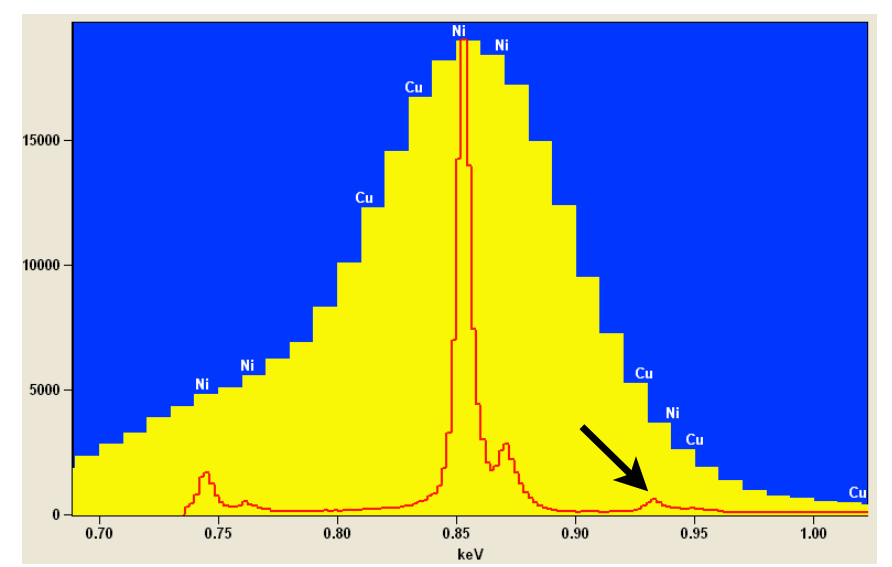

Figure 3. Identification of trace $\mathrm{Cu}$ in Ni. 450s acquisition time. Hitachi S-6600 FESEM.

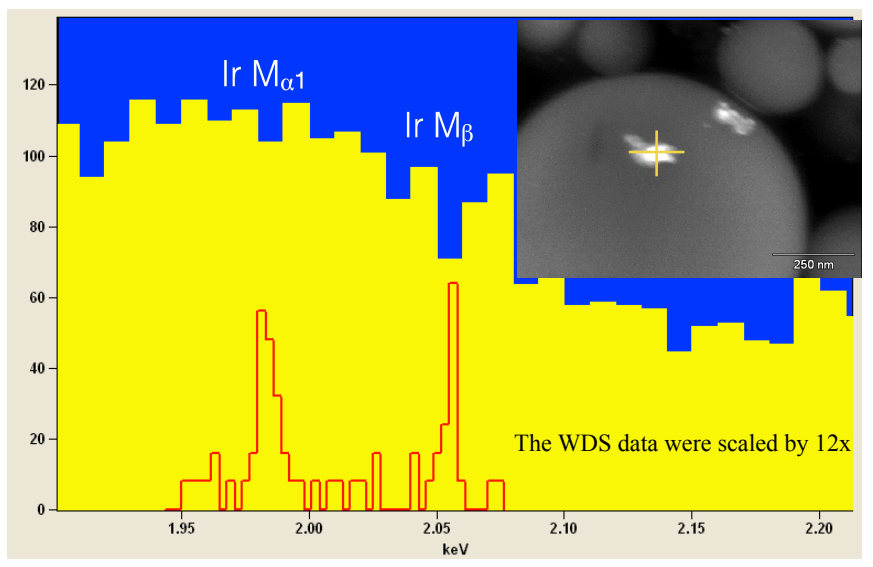

Figure 5. Identification of Ir nanoparticle. 22s acquisition time. Zeiss Merlin FESEM 